Int. J. Morphol.,

27(2):527-538, 2009.

\title{
Venas Superficiales de la Fosa Cubital. Aspectos Anátomo-Clínicos y Antropológicos
}

\author{
Superficial Veins of the Cubital Fossa. Anatomical, Clinical and Anthropological Aspects
}

\author{
"Mariano del Sol \& **Bélgica Vásquez
}

DEL SOL, M. \& VÁSQUEZ, B. Venas superficiales de la fosa cubital. Aspectos anátomo-clínicos y antropológicos. Int. J. Morphol., 27(2):527-538, 2009.

RESUMEN: Es bastante frecuente el acceso a las venas superficiales de la fosa cubital, siendo uno de los sitios más utilizados para punción venosa. Aunque es un procedimiento simple, es invasivo y a veces doloroso. La disposición de las venas superficiales de la región cubital ha sido descrita por numerosos autores y en diversos grupos étnicos, describiéndose variaciones y diversas padronizaciones. Las venas superficiales de la fosa cubital se han descrito formando una M, N, Y y W. Numerosos estudios, en distintas razas y grupos étnicos han demostrado similitudes y diferencias en la disposición de las venas superficiales de la fosa cubital. En 1908 Berry \& Newton determinaron que en el $83 \%$ de los hombres británicos la vena cefálica del antebrazo (VCA) y la vena basílica del antebrazo (VBA) eran conectadas por la vena mediana del codo (VMCo). Okamoto (1922) en hombres japoneses, determinó 3 Tipos de padrones venosos: Tipo I donde la VCA origina la VMCo, no existe la vena cefálica accesoria del antebrazo (VCAA), y la VCA no se duplica; Tipo II, caracterizado por la duplicación de la VCA y un Tipo III, que incluye la VCAA que drena en la VCA. En hombres blancos y negros Charles (1932) señaló que la distribución más frecuente (cerca de 70\% de los casos), era aquella donde la VCA y la VBA eran conectadas por la VMCo. Soller et al. $(1962,1964)$ en africanos de Africa Occidental, distinguiron tres tipos de formaciones venosas. Grupo I. Disposición clásica, tipos en M o aparentes (38,1\%). Grupos II y III descritas como disposiciones de tipo embrionario constituyen el $62 \%$ de los casos. Halim \& Abdi (1974) en hindúes, observaron tres tipos: 1) Tipo I. La VCA y la VBA son conectadas por la VMCo; Tipo II. La VCA drena en la VBA; la vena mediana del antebrazo (VMA) drena en la VCA. Tipo III. No existe comunicación entre la VCA y la VBA en la fosa cubital y la subdividen en Tipos III A y III B. Wasfi et al. (1986) describieron seis tipos de formaciones venosas en iraquíes, indicando que dos de ellas no habían sido mencionadas anteriormente. El tipo más común era la división de la VMA en dos venas, una de las cuales se unía a la VBA y la otra a la VCA. Del Sol et al. (1988) clasificaron las formaciones venosas de la fosa cubital en cinco Tipos: I la VCA se divide en vena mediana basílica (VMB) y vena mediana cefálica (VMC), uniéndose a la VBA y VCAA, respectivamente. Tipo II, la VCA origina la vena VMCo, que se une a la VB, no existe VCAA. Tipo III, no existe comunicación entre la VBA y VCA a nivel de la fosa cubital, Tipo IV, la VCA drena en la VBA y la VMA drena en la VCA. Tipo V otras disposiciones, donde se incluye la M clásica que resulta de la división de la VMA. Con respecto al diámetro de las venas parece ser de consenso que dependiendo del tipo de formación la VMB y la VMCo son las de mayor diámetro, La utilización de la VMC se recomienda cuando ella presenta un diámetro semejante a la VMB o a la VIMo ya que los riesgos de punción de otras estructuras anatómicas, como los ramos anteriores del nervio cutáneo medial del antebrazo o arteria braquial, son mínimos.

PALABRAS CLAVE: Anatomía; Venas; Fosa cubital; Punción venosa.

\section{INTRODUCCIÓN}

Las venas superficiales de la fosa cubital en el Hombre ha sido, durante mucho tiempo, un tema de interés para los anatomistas, morfólogos y antropólogos como también, para los profesionales del área de la salud quienes deben de manera constante intervenir en esta región del miembro superior.
Las venas superficiales de la fosa cubital son el sitio predilecto para la administración de soluciones parenterales, extracción o colocación de sangre y elementos terapéuticos e inclusive denudación venosa. Como fue mencionado por del Sol et al. (2007), en las últimas décadas las venas de la fosa cubital están siendo utilizadas en numerosas interven-

\footnotetext{
Facultad de Medicina, Universidad de La Frontera, Chile

** Universidad Autónoma de Chile, Temuco, Chile.
} 
ciones más especializadas e importantes como la introducción de catéteres en la obtención de sangre desde las cámaras cardiacas y para la realización de angiocardiografías, entre otros procedimientos.

La punción venosa, aunque se realice en las venas superficiales es invasiva, como lo señalan Yamada et al. (2008) y, por lo general, ligeramente doloroso, pero a veces provoca hemorragias internas y dolor crónico persistente, que se conoce como síndrome de dolor regional complejo. En la región cubital los ramos nerviosos superficiales provienen de los nervios cutáneo medial del brazo (ramo directo del fascículo medial del plexo antebraquial) y del nervio cutáneo lateral del antebrazo, uno de los ramos terminales del nervio musculocutáneo.

Las arterias normalmente se encuentran situadas profundamente a la fascia superficial y a los músculos. Sin embargo, en algunos sitios las arterias se encuentran sólo separadas de la piel por la fascia superficial. En otras ocasiones, arterias que normalmente son profundas, presentan un trayecto superficial subfascial como fue mencionado por Cagnon (1966) y del Sol et al. (1995, 2000), entre otros autores. Estas variaciones anatómicas adquieren importancia clínica, ya que pueden ser confundidas por venas, especialmente en los sitios más frecuentes de punción venosa, como la fosa cubital. Del Sol et al. (1995) reportaron un caso de arteria radial superficial bilateral a nivel de la fosa cubital. La arteria braquial se dividía en el tercio proximal del brazo, en las arterias radial superficial y ulnar. La arteria radial superficial se encontraba cubierta, a nivel de la fosa cubital, en ambos miembros, por un desdoblamiento de la aponeurosis bicipital que la separaba de la vena intermedia del codo (Tipo II) y del nervio mediano y arteria ulnar, localizados más profundamente.

Debido a los diversos procedimientos a que son sometidas las venas superficiales en la fosa cubital, ha llamado la atención de anatomistas y cirujanos, siendo objeto de numerosas investigaciones tanto en individuos adultos como en recién nacidos. En este último caso, el conocimiento anatómico de estas venas debería ser aún más importante ya que en niños muy pequeños, no es raro que se realizen intervenciones en áreas de mayor riesgo como, por ejemplo, la punción de las venas yugulares, femoral y otras. Incentivados por lo anterior, del Sol et al. (1988) realizaron un estudio de las formaciones venosas del miembro superior en recién nacidos, entre uno y doce meses de edad, observando el largo de las venas mediana basílica (VMB), mediana cefálica (VMC) y mediana cubital o mediana del codo (VMCo), como también la determinación de sus diámetros. Además, padronizaron las formaciones venosas de la fosa cubital con la finalidad de determinar el mejor sitio de punción venosa.
El objetivo de la presente revisión fue resumir brevemente las diversas descripciones que se han hecho de las formaciones venosas en la fosa cubital. Numerosos investigadores, anatomistas y cirujanos han descrito las diversas formas y distribución que presentan las venas en la fosa cubital en distintos grupos étnicos, países y regiones. Así, se pueden comparar y demostrar la existencia de innumerables variaciones venosas en la región cubital y que ha determinado su padronización por parte de algunos autores.

\section{TERMINOLOGÍA ANATÓMICA}

Las venas superficiales de la fosa cubital fueron consideradas en su totalidad en la última Nomina Anatomica, de Edimburgo el año 1989. Posteriormente, fueron retiradas de la Terminología Anatómica (2000) la VMB, VMC, dejando solamente a VMCo, vena basílica del antebrazo (VBA) y vena cefálica del antebrazo (VCA) y la presencia eventual de la vena cefálica accesoria del antebrazo (VCAA). Esta decisión fue tomada por indicaciones del Comité Federativo de Terminología Anatómica. Por lo anterior, la Terminología Anatómica no permite clasificar a las venas de la fosa cubital en el Tipo I, es decir en formación Tipo M (Clásica o no, descrita tanto por los anatomistas clásicos como los más recientes). Tanto la parte terminológica como de inclusión y exclusión de las venas de la fosa cubital en la nomenclatura internacional fue ampliamente comentada por del Sol et al. (2007). Estos mismos autores señalaron que no utilizaron la Terminología Anatómica actual porque ella dejó de lado la VMC y VMB elementos fundamentales de la división de la vena cefálica del antebrazo a nivel de la fosa cubital. Del Sol et al. (2007) usaron VI Nomina Anatómica aprobada por el INAC, el año 1985 en Londres y publicada en Edimburgo en 1989.

La nomenclatura anatómica utilizada en los diversos estudios para denominar las venas de la fosa cubital son muy diversas, lo que en algunos casos torna difícil su comprensión. Respetaremos la nomenclatura utilizada por los autores, sin embargo haremos uso además de la Terminología Anatómica (la cual está actualmente en uso y ha sido aprobada por los anatomistas en el mundo) a la cual incorporaremos la VMB y VMC.

\section{RESEÑA HISTÓRICA}

En la literatura médica mundial, se encuentran numerosas publicaciones sobre la distribución venosa superficial en la fosa cubital en el Hombre. En Chile se destacan los 
trabajos de Skewes (1944) en individuos chilenos de ascendencia hispánica y de del Sol et al., $(1990,2007)$ en jóvenes chilenos de diferentes grupos étnicos y en individuos mapuche.

Hace aproximadamente de 500 años, Andreas Vesalius de Bruxelas (1514-1564) escribía «De Humani Corporis Fabrica», libro que revolucionó el conocimiento anatómico de la época. En este libro, donde se mezcla la ciencia y el arte, es posible encontrar maravillosas ilustraciones anatómicas. En un diseño donde se representa de manera completa la vena cava, liberada de las partes anatómicas, se puede observar aspectos no habituales, como por ejemplo, la vena cava era considerada un vaso único y continuo, probablemente como también ocurre con la disposición no humana de las venas renales. Además, hay que considerar la influencia de Galeno o algunas concepciones de Aristóteles en los diseños de Vesalius. Sin embargo, este gran anatomista tenía un concepto bastante cercano a lo actual en relación a las venas de la región antebraquial anterior y fosa cubital. Puede ser observado en el grabado 44 una vena proveniente de la parte dorso lateral de la mano la cual asciende ventralmente en el antebrazo (VCA) y se divide en VMB y VMC, formando una $\mathrm{M}$ al unirse a dos venas paralelas que suben por la parte medial y lateral de la región antebraquial anterior, siendo éstas las VBA que se origina de la parte medial del dedo mínimo y VCAA que viene del extremo distal del dorso del antebrazo. Además, es posible reconocer venas que se originan en la palma de la mano y que en dos troncos terminan en la VMB y en la VBA, representando tal vez, a la vena mediana del antebrazo (VMA).

En un esquema Smith (1845) presenta la típica M clásica, con la división de la VMA en VMC y VMB además de la VCA y VBA. Llama la atención que la VMA se origine en el tercio inferior de la cara ventral del antebrazo. Este hecho ya se sabe que no es correcto, lo más probable que aún siendo Smith un anatomista americano, haya seguido la línea de los anatomistas franceses.

Jamain (1862) señaló que las venas superficiales del antebrazo son más numerosas en la región anterior del antebrazo que en la región posterior del mismo. Describe tres venas superficiales y paralelas la VR (VCA) que es continuación de la VC del pulgar, la VCubital (VBA) y la VMA, la ella señala que no es tan constante, como las dos venas precedentes y su diámetro está, por lo general, en razón inversa del de estos dos vasos. En ciertas circunstancias está representada por una red venosa superficial que establece comunicación entre la VCA y la VBA.

Aún con tal descripción anatómica Jamain indica que se observan cinco venas en la flexura del brazo o fosa cubital: VCA, VBA, VMA, VMC y VMB es decir una formación en
M clásica, lo que se contradice de cierta manera al señalar la inscontancia de la VMA.

En 1876 Sappey describió tres venas superficiales en el antebrazo: una anterior o VMA, una lateral o VCA y una medial o VBA; la VCA y la VBA según este autor pueden ser únicas o dobles. Estas venas en el pliegue del codo, adoptan, con mayor frecuencia, la forma de una $\mathrm{M}$, donde las ramas oblicuas son la VMC y la VMB, originadas de la bifurcación de la VMA, y las ramas verticales son la VCA y la VBA. Sin embargo, Sappey hace notar que esta disposición puede presentar numerosas variaciones, tales como, la ausencia de la VMA, en cuyo caso sus dos ramas son originadas por la VCA, o la ausencia de toda la parte mediana de este sistema venoso, en cuyo casos las venas radiale se unen para formar la VCA, y las venas ulnares convergen para formar la VBA. Similar descripción puede ser encontrada en los relatos de Testut (1894). Para este autor, la disposición de las venas superficiales del antebrazo es muy inconstante, siendo imposible enumerar todas la variedades. En algunos casos, la VCAA puede estar ausente, y con ella, en algunas ocasiones, también la VMC, reuniéndose todas las venas en un único tronco, la VBA, en el pliegue del codo. Se puede observar además, la duplicación de la VBA. Ya en 1902 Winslow describía este tipo de formación como la M clásica formada por la VMA, que se divide en VMC y VMB, por la VCA y por la VBA.

Tillaux (1900) describió tres gruesas venas que llegaban desde la región antebraquial anterior a la fosa cubital, VCA, VBA y VMA, esta última dividiéndose en las VMC y VMB formando la tradicional $\mathrm{M}$ venosas del codo. Como dato anecdótico Tillaux señaló «Las personas ignorantes, entre las cuales suceden más a menudo accidentes, son más propensos a puncionar la vena mediana basílica que es típicamente más grande y, por tanto, más accesible que la vena mediana cefálica».

Posteriormente, Bardeleben (1902), Bertelli (1902), Duval (1902), Poirier \& Charpy (1902) y Theile (1902), con algunas diferencias no significativas señalaron que la " $M$ " es la disposición más común, estando formada por la VCA y sus dos ramas (VMC y VMB), la VCAA y la VBA.

Poirier \& Charpy (1902) señalaron que existe a veces por medial una vena ulnar accesoria (VUA), y por la parte lateral una VCAA. Además, cuando existe una VMA, generalmente drena en la VCA cerca de su bifurcación, o en la VMB. Los sistemas venosos superficial y profundo se comunican a nivel del pliegue del codo a través de la vena comunicante del codo (VCCo), que drena en la VMC.

En 1908 Berry \& Newton describieron la distribu- 
ción venosa superficial de la zona cubital en 300 hombres británicos. Sus observaciones pueden resumirse de la siguiente manera: En un $83 \%$ de los casos, la VCA y la VBA fueron conectadas por la VMCo. En un $43 \%$ de estos casos la VMCo recibió a la VMA. En un $82 \%$ de los casos estuvo presente la VCAA. En un 16\% de los casos, la VCA y la VBA fueron conectadas por la VMC y la VMB, originadas de la bifurcación de la VMA. En el 1\% de los casos no había conexión entre la VCA y la VBA en la fosa cubital. Los autores describen asimismo, algunas variaciones anatómicas, entre ellas la duplicación de la VMCo (4\%), la conexión entre la VCA y la VCAA en dos casos; la ausencia de la VMCo, la VMA y la VCAA (cuatro casos) y la duplicación de la anastomosis entre la VCA y la VBA por medio de la VMC y la VMB (seis casos). En cuatro casos, la VCA no originaba la VMCo, estando esta última representada por la porción terminal de la VMA. En un caso la VCA drenaba en la VBA y en cuatro casos la VMA estaba ausente.

Chiarugi (1912) refirió que la VCA originaba en el pliegue del codo la VMCo, que se dirigía hacia la VBA. Con mucha frecuencia, sobre el origen de la VMCo, la VC recibía a la VCAA. Por otra parte, las VvMA terminan en la fosa cubital, la mayoría de las veces en la VMCo o en la VBA, raramente en la VCA. La VMCo recibe a la VCCo.

Una observación interesante efectuó Chiarugi, la disposición plexiforme que presentan las venas superficiales de la región antebraquial anterior. Señaló por otra parte que, el tronco principal de recolección es la VCA o VBA, el cual inicialmente tienen un trayecto sobre la cara posterior del antebrazo para luego alcanzar la cara anterior del mismo continuando en el brazo para colocarse en los surcos bicipitales lateral y medial. Otros troncos venosos colectores menores son la VMA y la VCAA.

Es conveniente destacar a Chiarugi quien fue uno de los primeros anatomistas en discrepar sobre la disposición clásica que adoptaban las venas en el antebrazo y en la fosa cubital en el hombre. Este autor señaló que no es la VMA la que origina la VMC y la VMC, sino que éstas son producto de la división de la VCA.

Diez años después, en 1922, Okamoto describió las venas de la fosa cubital en 200 hombres japoneses, determinando los siguientes padrones venosos: Tipo I donde la VCA origina la VMCo, no existe la VCAA, y la VCA no se duplica, distribución que encontró en 19\% de los casos ( $21 \%$ en el lado derecho, $17 \%$ en el lado izquierdo); Tipo II, caracterizado por la duplicación de la VCA, observado en $27 \%$ de los casos (31\% en el lado derecho, $23 \%$ en el lado izquierdo), y un Tipo III, que incluye la VCAA que drena en la VCA y que fue encontrado en el $43 \%$ de los casos (47\% en el lado derecho, $39 \%$ en el lado izquierdo).

Para Okamoto la VBA es un vaso de curso y terminación constantes, sólo observó dos variaciones de esta vena. En una de ellas, al VBA se duplicó; en la otra, la VBA era muy delgada, siendo en compensación muy gruesa la VCAA. El autor observó la VMCo en un 85,5\% de los casos. En un $54,5 \%$ de los casos esta vena drenaba en la VMC y en un $8,5 \%$ era doble. En $14,5 \%$ de los casos la VMCo no estaba presente. La VMA terminaba en el $44 \%$ de los casos en ambos lados en la VBA; en 54,5\% terminaba en la VMCo; en dos casos terminaba dividiéndose en VMC y VMB (distribución en M), o bien terminaba en un ostio en la vecindad de la VMCo. Este ostio se abría en la VMCo o en la VBA, en el $13 \%$ de los casos.

Por otra parte, Okamoto señaló algunas anormalidades en la distribución venosa superficial del antebrazo. Así en el 8,5\% de los casos la VMCo era doble y en el 14,5\% esta misma vena estaba ausente. Además, hubo conexión entre la VCA y la VCAA en un 2,5\% de los casos y entre la VCA y la VMA en un 4,5\% de los casos. En un caso, el autor observó la presencia de dos ostios en la región de la fosa cubital, en los cuales drenaron las VvMA.

Según Rouvière (1924) las redes venosas de la mano dan origen a tres troncos principales, que son los troncos colectores de las venas principales del antebrazo, denominados vena radial superficial (VRS) o VMA, vena cubital superficial (VCuS) y vena radial del antebrazo (VRA). La VRS se divide en el pliegue del codo en VMC y VMB. A este nivel recibe a la VCCo. La VCuS se une a la VMB para formar la vena basílica (VB) y la VRS se une a la VMC para formar la vena cefálica (VC). Así para este autor las VRS, VRA, VMC, VMB y VCuS, constituyen una M venosa en la fosa cubital.

Si se analiza la ilustración de Rouvière en la cual representa las venas de la fosa cubital, se puede observar que la denominada VRS o VMA no es otra que la VCA ya que proviene de la VC del pulgar y luego asciende hacia medial para colocarse ventral en el antebrazo; luego se divide en las VMC y VMB. La VCuS correspondería a la VBA ya que se origina de la vena colateral medial del dedo mínimo (salvatela), siguiendo por la parte medial de la cara anterior del antebrazo, se une a la VMB, y por la VRA (VCAA) que viene de la cara posterior del antebrazo, contornea el margen lateral hacia el límite inferior del pliegue cubital y se unía por encima del epicóndilo lateral a la VMC. Este mismo autor no representa a la VMA, lo que concuerda con muchas observaciones y que ratifica la poca importancia de esta vena en la formación de las venas de la fosa cubital. 
Para Tandler (1929), las variedades de las venas superficiales en la fosa cubital son muy numerosas, pero pueden describirse varios tipos fundamentales. Señala que se encuentra con frecuencia una rama anastomótica que cruza oblicuamente la región de la fosa cubital, la VMCo, que está separada de los vasos profundos del brazo por la aponeurosis bicipital. Una importante rama colateral la pone en comunicación con las venas braquiales profundas. La VMA dessemboca en este caso generalmente en la VMCo. Según este mismo autor, otra variedad que se observa muy frecuentemente consiste en que la VMA se divide en dos ramas, de las que la más débil se abre en la VC es la VMC, mientra la otra, designada VMB, aboca a la VB.

Con respecto a las formaciones venosas en la fosa cubital nos parece interesante el relato que hace Tandler sobre la VMA. Este autor señala que la VMA en la cara ventral del antebrazo se extiende entre las dos importantes venas que siguen por ambos bordes, la VCA y la VBA, una red vascular de anchas mallas, de la que sobresle un tronco longitudinal que se dirige a la mitad del codo. «Tan variable como la existencia de este vena es su punto de abocamiento». Sin embargo, precisamente el último segmento de la VMA es de gran interés práctico. Ya en esos años Tandler recalcaba que, las venas del codo, debido al escaso desarrollo del panículo adiposo de la región, son las venas más voluminosas, más accesibles al cirujano, por cuyo motivo eran utilizadas para la práctica de la sangría y de las inyecciones intravenosas.

En unartículo publicado por Charles en 1932, realizado en las venas de la fosa cubital de 122 cadáveres de hombres blancos y negros encontró que la distribución más frecuente (cerca de $70 \%$ de los casos), era aquella donde la VCA y la VBA son conectadas por la VMCo. La segunda distribución más frecuente $(27,5 \%)$ en los blancos americanos, la VCA y la VBA se conectan en forma de M, con la VMA, la VMB y la VMC. Este tipo se observó con menor frecuencia en los negros americanos (12,1\%). En los negros, la segunda distribución más frecuente $(14,5 \%)$ es aquella en que no existe comunicación entre la VCA y la VBA en la fosa cubital, disposición que fue observada por el autor solamente en el lado izquierdo de los blancos americanos $(3,3 \%)$. En cerca del 15\% de los casos, el autor observó la duplicación de la VCA, lo que ocurrió con similar frecuencia en los dos grupos estudiados. La VMA terminaba en forma más frecuente (cerca del 45\%) en la VMCo, en los dos grupos, excepto en el lado izquierdo de los individuos blancos americanos $(3,3 \%)$.

Por otra parte, Charles señaló que en cerca del $15 \%$ de los casos, la VCA estaba duplicada, lo que ocurrió con similar frecuencia en los dos grupos estudiados. La VMA terminaba en forma más frecuente (cerca del $45 \%$ ) en la VMCo, en los dos grupos, excepto en el lado izquierdo de los negros americanos. En aproximadamente el 35\%, la VMA drenaba en la VBA. La bifurcación de la VMA en VMB y VMC fue observada con mayor frecuencia en blancos americanos $(27,5 \%)$, que en negros americanos $(12,1 \%)$. La presencia de una VMCo accesoria fue observada con mayor frecuencia en blancos americanos $(4,2 \%)$ que en negros americanos $(0,8 \%)$.

Las principales venas superficiales del antebrazo según Salvi (1932) son la VBA, VCA y las VvMA, pudiéndose encontrar también la VCAA. La VCA origina la VMCo, que drena en la VB del brazo. Cerca de su origen, se desprende de la VMCo, la VCCo, pudiendo esta última rama ser originada también de la VCA antes de su bifurcación. En el codo la VCA puede recibir a la VCAA.

Para Salvi las VvMA terminan en la VMCo, a distintos niveles, o bien drenan en la VCA, pero una de ellas, la más cercana al lado ulnar y la de mayor diámetro, drena en la VBA antes que ésta reciba a la VMCo. Según este autor, la distribución de estas venas presenta variaciones: la VMCo puede ser muy delgada, puede duplicarse o puede estar ausente; las VvMA tienen un tipo de drenaje muy variable, pudiendo en ausencia de la VMCo, reunirse en dos ramas, una que drena en la VC del brazo, y la otra en la VB del brazo. Par este autor, cuando existe la VCA, se observa en el pliegue del codo una disposición venosa en forma de $\mathrm{M}$, o de una Y rodeada de dos canales longitudinales paralelos. La VCA al dividirse en la fosa cubital origina las dos ramas mediales; las ramas verticales son la VCAA y la VBA. Finalmente, este autor afirma que la VMA de otros autores corresponde en realidad a la VCA, que la VCA es la VCAA y que la VMC es la VCA.

Uno de los primeros en estudiar las distribuciones venosas en la fosa cubital en chilenos fue Skewes en 1944, quien en 140 individuos de sexo masculino observó lo siguiente: La VMCo conectaba al VCA y la VBA en un $78 \%$ de los casos. En un $84,35 \%$ de los casos estaba presente la VCAA, observándose una disposición en M. La VMA estuvo presente en el 83,15\% de los casos; en un 66,1 drenaba en la VMCo; en un $32 \%$ drenaba en la VBA y en 1,7\% de los casos se dividía en VMC y VMB (M clásica). Finalmente, señaló que en el 2,4\% de los casos no había conexión entre la VCA y la VBA en la fosa cubital. Para este autor, la VBA es la vena más constante del miembro superior $(100 \%$ de los casos), en tanto que la VCA estuvo ausente en dos casos $(1,7 \%)$.

Paturet en 1951 describió cuatro tipos de formaciones venosas en la fosa cubital: 1) Tipo en M. El más fre- 
cuente (60\% de los casos). La M está formada por medial por la VMB, por lateral por la VMC y la distribución lateral, corresponde a la VRS por lateral y la VCuS por medial. En la punta de la M se agrega la VMA. También, en ocasiones, se agrega una VRA y la VCCo. La VMC es generalmente más delgada y superficial que la VMB. 2) Tipo en Y. Es bastante frecuente (30\% de los casos). Esta representado por la VRS, que se continúa por la VMB y por la VMC, generalmente delgada. En este tipo existe casi siempre una VRA, que drena en el origen de la VC del brazo. La VMA, generalmente delgada, drena en la VMB. C) Tipo en N. Para el autor, este tipo es poco frecuente ( $8 \%$ de los casos). La VRS se bifurca en dos troncos de igual volumen, uno lateral (VC) y otro medial, la vena oblicua del pliegue del codo (VOPC). En este tipo, no existe la VMC y la VMA drena en la VOPC, por encima del origen de la VOPC bajo la VCuS. D) Tipo en W. Se encuentra en sólo $2 \%$ de los casos. Se caracteriza por la ausencia de la VMA, por una duplicación de la VBA y por una bifurcación relativamente alta de la VRS. La división de la VRS da como resultado una VMC corta y delgada y una VMB larga y voluminosa. En este tipo, los brazos de la W están representados por la VMC y VMB (ramas mayores) y por las dos ramas de bifurcación de la $\mathrm{VCuS}$ (ramas menores).

Orts Llorca (1952) describió que la VC, a nivel de la fosa cubital, emite una voluminosa rama anastomótica, que se une a la VBA, siendo en la mayoría de los casos la VMCo voluminosa y la VC, arriba de ella, muy fina. Con frecuencia la VMA se divide en VMB y VMC.

En dos figuras diferentes Pernkopf (1953) presenta: 1. División de la VMA en VMC y VMB, uniéndose a la VCA y la VBA, respectivamente; 2. VCA dividiéndose en VMC y VMB o VMCo, las cuales se unen a la VCA y a la VBA, respectivamente. En este último caso, la VMA drena en la VBA. Además de esto, describe la formación en Y.

Sohier et al. (1962 y 1964), realizaron 55 disecciones de la fosa cubital en africanos de Africa Occidental, distinguiendo tres tipos de formaciones venosas. Grupo I. Disposiciones clásicas, tipos en $\mathrm{M}$ o aparentes en un 38,1\% de los casos. Grupos II y III descritas como disposiciones de tipo embrionario. Constituyen el $62 \%$ de los casos. En el grupo II, hay predominancia considerable de la rama cubital. La VBA colecta la casi totalidad de la sangre venosa superficial. Fue observado en 25,4\% de los casos. En el grupo III, hay una separación casi completa de las dos ramas, siendo preponderante la rama basílica en el $36,4 \%$ de los casos.

De la misma manera que el caso anterior, Halim \& Abdi en 1974 estudiaron la distribución de las venas de la zona cubital en 536 sujetos hindúes (136 vivos y 400 cadá- veres), clasificando sus observaciones en tres tipos: 1) Tipo I. La VCA y la VBA son conectadas por la VMCo; puede o no estar presente la VCAA. Fue observado en 362 casos (67,5\%); en 9 de ellos, la VMCo estaba duplicada. La VMA drena en la VMCo, en la VBA o en la VCA. 2) Tipo II. La VCA drena en la VBA; la VMA drena en la VCA. Este tipo fue observado en 105 casos (19,5\%). La porción braquial de la $\mathrm{VC}$ estuvo ausente o era rudimentaria, en 26 casos (4,9\%), o fue reemplazada por una VCA, en 78 casos (14,5\%). 3) Tipo III. Este tipo, en el que no existe comunicación entre la VCA y la VBA en la fosa cubital, fue observado en 67 casos $(12,5)$. Los autores lo subdividen en un Tipo III A, que fue observado en el 6\%, y un Tipo III B, encontrado en 6,5\%. En el Tipo III A, la VMA drenó en la VCA (5 casos) o en la VBA (22 casos), o se duplicó, drenando una de las ramas en la VCA y la otra en la VBA. En el Tipo III B, la VMA se dividió en VMC y VMB, que drenaron en la VCA y la VBA, respectivamente.

Para Warwick \& Williams (1979), la VCA origina la VMCo, que drena en la VBA y que recibe una rama comunicante desde las venas profundas del antebrazo. Bajo el pligue del codo, la VCA recibe a la VCAA. La VMA drena en la VBA o en la VMCo, o puede dividirse en dos ramas, una para la VBA y la otra para la VCA.

Según Fumagalli \& Cavallotti (1982), la VR o VCA se une en el pliegue del codo a la VMC para desembocar en la VC y la vena ulnar superficial (VUS) o VBA drena, uniéndose a la VMB, en la VB del brazo. La VCCo drena en la bifurcación de la VMA, en la VMB o en la VMC.

Hamilton (1982) señaló que por delante del codo existe una larga VMB, que se dirige desde la VCA hacia la VBA. Ocasionalmente, se observa una gran VMA que se divide en VMC y VMB, que se separan para formar las VC y VB, respectivamente.

Moore (1982) describió a las venas de la fosa cubital formando una M, en la cual la VMA se divide en dos ramas, una denominada VMCo, y la otra sin denominación, que se reúnen en la VB y VC, respectivamente. Los brazos laterales de la M están constituidos por la VCA por lateral y la VBA por medial. Las venas profundas se conectan con la VMCo a través de la VCCo.

En 1983 Latarjet \& Ruiz Liard vuelven a indicar que las venas superficiales de la fosa cubital a este nivel dibujan la $\mathrm{M}$ venosa del pliegue del codo. Señalan además que la «vena media» recibe aquí una anastomosis constante de la red profunda, la vena perforante del codo.

Snell (1984) describe tres tipos más frecuentes de dis- 
tribución venosa en la fosa cubital. En el primer tipo, la VMCo conecta la VC y la VB y la VMA drena en la VMCo. En el segundo tipo, la VMCo conecta la VC y la VB y la VMA drena en la VB. En el tercer tipo, la VMA se divide en VMB y VMC, las que se unen a la VB y VC, respectivamente.

Para Woodburne (1984), la VCA está unida en la parte frontal del codo a la VBA por medio de la VMCo, que drena en la VB, y que está relacionada con las venas profundas del antebrazo. A nivel del codo, la VCA recibe a la VCAA. La VMA termina en la VMCo o en la VMB; algunas veces se divide en $\mathrm{VMB}$, que en trayecto y terminación sustituye a la VMCo, y en VMC, la cual se une a la VC. En tales casos, las venas de la fosa cubital muestran una distribución en forma de M. En ocasiones, la VMA puede estar ausente.

En un artículo sobre la disposición de las venas superficiales de la fosa cubital realizado en 300 iraquíes Wasfi et al. (1986) describieron 6 tipos de formaciones venosas, indicando que dos de ellas no habían sido mencionadas anteriormente. Estos autores señalaron que el tipo más común era que la VMA se dividía a nivel de la fosa cubital en dos venas, una de las cuales se unía a la VB y la otra a la VC. Además, indicaron que esta división podía producirse en pocos casos incluso proximal a la fosa cubital. También señalaron que la disposición no reportada anteriormente era la comunicación entre las VBA y VCA a través de una conexión venosa horizontal entre una de las tributarias de las dos venas y la VB y que la VMA se divide en VMB y VMC y una vena de la parte anterior del antebrazo drena en la VMB. Sin embargo, estas dos variaciones anatómicas mencionadas por Wasfi ya habían sido descritas e incluso aparecen en figuras de diversos tratados de Anatomía.

En el año 1988 del Sol et al., estudiaron las venas superficiales de 40 antebrazos de niños entre 0 y 1 año, de ambos sexos, clasificando las formaciones venosas de la fosa cubital en cinco tipos:

Tipo I. La VCA se divide en VMC y VMB, uniéndose a las VC y VB, respectivamente. La VMA drena generalmente en la VMB. Los autores clasificaron 12 casos (30\%) dentro de este tipo. En 9 de estos 12 casos (75\%), la VMA drenó en la VMB; en un caso lo hizo en la VMC y en tres casos, la VMA estaba ausente.

Tipo II. La VCA origina la VMCo, que se une a la VBA. No existe la VCAA. Encontraron 12 casos (30\%). En cuatro formaciones venosas no estaba presente la VMA; en siete casos la VMA drenaba en la VMCo y en un caso lo hizo en la VBA. En un caso la VMCo era doble.
Tipo III. En este tipo no existe comunicación entre la VCA y la VBA a nivel de la fosa cubital. Se encontró en10 casos (25\%). En 8 de esos casos no existía VMA; en los otros dos casos, esta vena drenó en uno de ellos en la VBA y en el otro caso, en la VCA.

Tipo IV. La VCA drena en la VB y la VMA drena en la VCA. Este tipo fue observado en cuatro casos (10\%). En dos de ellos la VMA estaba ausente; en los otros dos, la VMA drenó en la VCA.

Tipo V. Otras disposiciones. Encontraron solo dos casos (5\%).

En el 100\% de los casos del Sol et al. (1988), observaron la vena perforante del codo, siendo en tres casos doble.

En otro estudio realizado por del Sol et al. (1990) analizaron las venas superficiales de la fosa cubital en 200 jóvenes chilenos de ambos sexos, de diferentes grupos étnicos, entre 17 y 24 años de edad. Basados en la clasificación de del Sol et al. (1988) obtuvieron los siguientes resultados: Tipo I en 36,25\%; Tipo II en $28,75 \%$, Tipo III en $17,25 \%$; Tipo IV en 14,75\%; Tipo V en 3\% de los casos. Sólo en el $0,5 \%$ observaron la M clásica de Winslow.

Para del Sol \& Olave(1991), la VIB se presenta más voluminosa en el Tipo I, siendo la VICo más voluminosa en el Tipo II. Indican estos autores que resulta más fácil la punción de estas venas, pero se debe tener presente que, relacionadas con ellas, se encuentran los ramos del nervio cutáneo antebraquial medial, los cuales en mayor número y grosor cruzan, principalmente, por su parte anterior y cuando son puncionados accidentalmente, provocan un dolor más agudo que en otras zonas de la fosa cubital. Además, señalan que otro de los inconvenientes para el abordaje de estas venas, es la relación que tienen, con la arteria braquial y el nervio mediano, estructuras de las cuales se encuentran separadas solamente por la expansión bicipital. Sugieren extremar el cuidado al efectuar la intervención en la fosa cubital. Finalmente indican que la utilización de la VMC o de la VC cuando ellas presentan un calibre semejante a la VMB o VMCo es recomendable, principalmente, porque los riesgos de punción arterial o nervioso son menores, no sólo porque el nervio mediano se encuentra a gran distancia, sino también porque el nervio cutáneo lateral del antebrazo, ramo terminal del nervio musculocutáneo, es retrovenoso.

En 1993 Queiroz vuelve a señalar que las venas superficiales del antebrazo y de la fosa cubital son tres: una mediana, una lateral y otra medial. La VMA según este autor recoge la sangre de la parte central de la red palmar y 
tiene su origen en la muñeca. Asciende luego verticalmente por la cara anterior del antebrazo hasta el pliegue del codo, donde se divide en dos ramas. La medial VMB que corre por encima del epicóndilo medial del húmero y se anastomosa con la «VCS» para formar la VB. La lateral, VMC se deliza por el borde lateral del músculo bíceps braquial y a la altura del epicóndilo lateral se une a la «VRS» para formar la VC.

Si se analiza el nacimiento de la «VRS» descrita por Queiroz, quien señaló que este vaso se origina por la unión del arco venoso dorsal con la vena cefálica del pulgar y luego asciende por el dorso del antebrazo, rodenado en su parte media el margen lateral pasando a la cara anterior. Corre por esta cara hasta el epicóndilo lateral, donde se une con la VMC para formar la VC del brazo. Como puede ser observado el trayecto descrito por Queiroz pareciese ser el recorrido por la VCAA, sin embargo, ésta no se origina de la VC del pulgar sino un poco más posterior en la muñeca. Por otra parte, quien nace de la $\mathrm{VC}$ del pulgar o de la unión con ella es la VCA, la cual daría la VMC o la VMCo dependiendo de la existencia de la VCAA. De cualquier manera, la descripción de Queiroz de las venas superficiales de la región cubital corresponde a la «M» clásica, señalada por el autor como una «M» mayúscula, pero como ya ha sido mencionado, la probabilidad de su existencia es rara.

Del Sol et al. (2007) estudiaron las venas superficiales de la fosa cubital en 300 miembros superiores de individuos de ambos sexos ( 30 hombres y 120 mujeres), chilenos, del grupo étnico mapuche, con edades entre 15 y 84 años. Basados en la clasificación de del Sol et al. (1988) obtuvieron los siguientes resultados: Tipo I en 38,7\%; Tipo II en 28,3\%, Tipo III en 24,0\%; Tipo IV en 4,3\%; Tipo V en 4,7\% de los casos. Los diámetros mayores de las venas en el Grupo I fueron la VMB $(41,4 \%)$ y la VMC $(28,4 \%)$; en el Grupo II fue la VMCo $(77,6 \%)$. La frecuencia de la VMA en Grupo I fue de 75,9\%, bajando al 69,2 \% en el Grupo III y al $50 \%$ en el Grupo II. La VMA drenaba en el $72,2 \%$ en la VBA en el Grupo I y en el 42,9 en la VMCo y VCA en el Grupo II y en el $75 \%$ en la VB en el Grupo III.

Como se puede recnocer en los diversos estudios de las venas superficiales de la fosa cubital, ellas presentan innumerables variaciones. Una de las principales características es la ausencia de una VMA que llega a dividirse o no en VMB y VMC. Esto ha quedado demostrado ya en estudios efectuados por Sappey, Wooburne, del Sol et al., 1988, 1990, 2007. Sin embargo autores como Skewes han reportado la ausencia de la VMA en los chilenos de ascendencia hispánica en un porcentaje bastante bajo (16,5\%). Más aún, para Paturet y Berry \& Newton la ausencia de la VMA es muy rara. Para Singh en nigerianos, del Sol et al. (1988), en re- cién nacidos brasileños y del Sol et al. (2007) la ausencia de la VMA es superior al 40\%. En consecuencia, es difícil sostener que la VMA sea la principal vena que drena la parte superficial de la región antebraquial anterior y sea la que termine dividiéndose en la VMB y VMC. Como lo demuestran numerosos autores, principalmente en sus esquemas es la VCA la que terminaría dando esta división.

En las formaciones venosas descritas se observa una tendencia hacia la formación de una $\mathrm{M}$ o de una $\mathrm{N}$, dependiendo de la existencia de la VCAA. Este tipo de formación, definida por del Sol et al. (1988) como Tipo I, es la más frecuente y está constituida por la VBA (medialmente), VCAA (lateralmente) y VCA (localizada entre ambas); las dos ramas terminales de esta última (VMB y VMC) se unían a la VBA y VCA, respectivamente. Este tipo de formación a nivel de la fosa cubital es bastante similar a lo reportado por Poirier \& Charpy; Bardeleben; Bertelli; Duval; Theile; Berry \& Newton; Chiarugi; Okamoto; Rouviére; Salvi et al.; Skewes; Warwick \& Williams; Woodburne; del Sol et al. (1988); del Sol \& Olave (1991) y del Sol et al. (2007).

Llama la atención que aún hoy día se mencione que la VMA sea la que se divide en la VMB y VMC a nivel de la fosa cubital. Esta "M" clásica descrita por Testut (1894); Winslow; Paturet en el 60\% de los casos; Singh et al. (1982) en el $40 \%$ en nigerianos; Sappey; Tandler; Berry \& Newton en el $16 \%$ en británicos y Charles en el $19,7 \%$ en americanos blancos y negros. Esta "M" clásica se presentó en el 1\% de los japoneses (Okamoto), igual cifra en individuos mapuche (del Sol et al., 2007), en el 1,7\% en chilenos de ascendencia hispánica (Skewes) y en el 6,5\% en hindúes (Halim \& Abdi, 1974).

Volvemos a ratificar que la " $\mathrm{M}$ " clásica de Winslow es un error conceptual y anatómico, en el sentido que algunos autores consideraban, y aún consideran, erróneamente la "Y" formada por la bifurcación de la VMA en VMC y $\mathrm{VMB}$, lo que creemos es una confusión, ya que la VMA debiese corresponder a la VCA y la denominada VCA, a la VCAA. Salvi, Chiarugi y del Sol et al. (1988 y 1990, 2007) ya señalaron este error. Sin embargo, nos parece que Salvi no estuvo en lo correcto al plantear que la VMB corresponde a la VMCo y que la VMC corresponde a la VCA. Para nosotros, al igual que para del Sol et al. (2007) esto sólo ocurre en caso de ausencia de la VCAA determinado una formación Tipo II.

Las formaciones venosas en la fosa cubital, Tipos I y II son las más frecuentes. Así lo demuestran los estudios de Charles $(27,5 \%)$ en americanos blancos y negros, del Sol et al. (1988), en el 30\% de recién nacidos brasileños, Paturet (30\%), del Sol et al. (1990, 2007), en el 36,5\% de los jóve- 
nes chilenos y en el 38,7\% en individuos mapuches, respectivamente. Llama la atención que la formación Tipo I se presente con mayor frecuencia en el miembro superior derecho, independiente del sexo, tal vez por la presencia de individuos diestros, como lo señalaran del Sol et al. (2007).

En general puede ser observado que la VMB, considerando ambo sexos y ambos lados, es la de mayor diámetro hecho señalado por del Sol et al. (1988) en recién nacidos brasileños y por del Sol \& Olave en los estudios realizados en jóvenes chilenos. Autores como Bardeleben; Bertelli, Duval y Thiele también indican a la VMB como la de mayor diámetro, pero para estos mismos autores la VMB es un brazo de la VMA. Por otra parte, la VMA está más desarrollada en los hombres que en las mujeres debido, posiblemente, a la mayor masa muscular y a la actividad física realizada.

Existen diferentes criterios en cuanto al drenaje de la VMA a nivel de la fosa cubital. Según del Sol et al. (1988 y 1990) esta vena en el Tipo I drena, generalmente, en la VMB. Sin embargo, Lagos \& Torres (1991) señalaron que la VMA drenaba con mayor frecuencia en la VBA $(43,8 \%)$ de los casos, seguido de la VCA en el $35,1 \%$, estudio realizado en individuos mapuche.

Cuando no existe la VCAA y la VCA del antebrazo da una rama hacia proximal y medial (VMCo), señalamos que se trata de una formación venosa Tipo II. Esta formación se presenta en general, entre un 20 al 30\%. Así, del Sol et al. (2007) la observaron en el $28,3 \&$ de los individuos mapuches; del Sol et al. (1988) en el 30\% de los recién nacidos brasileños; del Sol et al. (1990) en el 28,75\% en jóvenes chilenos. Muy elevados porcentajes de formaciones venosas Tipo II en la fosa cubital fueron señalados por Berry \& Newton en el $83 \%$ de los británicos y por Charles, en el $70 \%$ de los americanos blancos y negros y bajos porcentajes por Paturet (8\%) y por Singh et al., en el $11 \%$ de los nigerianos. En definitiva pareciera ser que este Tipo II de formaciones venosas en la fosa cubital es común. Aún más, considerando que la VCAA es relativamente pequeña en comparación con la VCA y la VBA algunos autores no deben considerarla y por tanto el porcentaje de formaciones venosas Tipo II sería el de mayor frecuencia. Por lo anterior es entendible que diversos autores como Warwick \& Williams, Snell, Charugi, Ort LLorca, Tandler y Salvi, entre otros señalen que esta formación es común o normal.

La formación de la $\mathrm{M}$ venosa en la fosa cubital, debido a la presencia de la VCAA es muy frecuente, $82 \%$ en los australianos según Berry-Newton; 75-80\% en finlandeses y suecos según Lassila (1931); 43\% en japoneses según Okamoto y $84 \%$ en chilenos según Skewes.
Autores como Berry \& Newton, Okamoto y Halim Abdi, han considerado los Tipos de formaciones venosas de la Fosa cubital I y II, como un único tipo, negando la existencia de la VMB y la VMC, lo que no justificaría la inclusión de estas venas en la Nomina Anatomica, situación que nos parece errónea. De esta manera, existiría solo la VMCo a nivel de la fosa cubital.

En las formaciones venosas Tipo II, la VCA otorga la VMCo, la cual es la de mayor diámetro y por consiguiente de mayor flujo. Esto se da independiente del sexo, grupo étnico, no existiendo diferencia significativa como lo señalan Lagos \& Torres (1991) en los individuos mapuche. Autores como Chiarugi han señalado que la VBA es más voluminosa que la VCA y que la VMCo es una gran rama de la VCA. Empero, se tiene que considerar tanto para la formación venosa Tipo I como para la Tipo II, ya que este autor no efectuó esta clasificación. Similares observaciones fueron realizadas por Orts LLorca en relación a que la VMCo se torna voluminosa, y después la VCA prosigue en el brazo como una pequeña vena. Al igual que en el Tipo I, en el Tipo II la VMA drena en la VBA, con mayor frecuencia.

En el Tipo III, no existe comunicación entre la VCA y VBA a nivel de la fosa cubital, se presentó según del Sol et al. (2007) en el 24\% de los individuos mapuche, sólo en el $1 \%$ de los británicos ( Berry \& Newton), en el $10 \%$ de los nigerianos (Singh et al.), en el $25 \%$ brasileños del Sol et al. (1988), en el 17,25\% de los jóvenes chilenos, del Sol et al. (1990), en el 12,5 en hindúes (Halim \& Habdi). Estos últimos autores, subdividen el Tipo III, en el Tipo III-A(6\%) y en el Tipo III-B o "M" clásica (6,5\%). La VMA cuando existe, en general drena en la VBA.

Para Sohier et al. (1964, 1969), la disposición de tipo embrionario, definida por ellos como Tipo II y Tipo III, ocurre en la mayoría de los casos (62\%) en las venas superficiales del pliegue del codo, en africanos de África Occidental. Sin embargo, hay que señalar que estas disposiciones serían semejantes al Tipo IV, pero con diferencias, ya que para el Tipo II, la VBA es predominante y recoge casi la totalidad de la sangre del antebrazo, drenando en ella una gran VMA y siguiendo por el brazo una pequeñísima VC.

Cuando la VCA cruza medial y proximalmente la parte superior del antebrazo y región cubital alcanzando la VB y terminando en ella (Tipo IV) existen variadas informaciones así del Sol et al. (2007) señalan su presencia en sólo el $4,3 \%$ de lo individuos mapuches, inferior a lo observado por Halim \& Abdi (19,5\% en individuos hindúes) y por Singh et al. (10\% en nigerianos). Por otra parte, Charles encontró esta formación en el 1,6\% de individuos americanos blancos y negros. Cabe destacar, la ausencia de este tipo de for- 
mación venosa en los individuos japoneses (Okamoto). Por otra parte, la VMA presente en el $61,5 \%$, de los individuos mapuches drenaba en el $100 \%$ de los casos en la VC, lo que resulta lógico desde el punto de vista anatómico ya que la VC se dirige hacia medial y hacia el brazo, alcanzando a la VB antes de que ésta perfore la fascia y transcurra profunda por el resto de su recorrido.

Las formaciones venosas (Tipo V), son todas aquellas que no encuandran en la clasificación de del Sol et al. (1988), incluida la "M" clásica descrita por Winslow y que para Chiarugi no existe. Esta formación representa solo el $1 \%$ de los individuos mapuche, por tanto debe ser considerada como una variación anatómica. No obstante lo anterior, la "M" clásica para Testut y otros autores es una formación común, siendo muy frecuente para Tandler y Orts Llorca. La "M" clásica, es extremadamente infrecuente según Poirier \& Charpy, Salvi, Chiarugi, Charles, Halim \& Abdi y del Sol et al. $(1988,1990,2008)$ y es la formación más común para Sappey, Winslow, Testut, Paturet, Singh et al., entre otros.

\section{CONCLUSIONES}

Es de vital importancia, antes de efectuar una punción venosa en la fosa cubital, analizar adecuadamente la disposición de estas venas, conocer los padrones más frecuentes, evitando de esta manera complicaciones como el síndrome de dolor regional complejo.

Las venas superficiales de la fosa cubital mantienen estrechas relaciones con otras estructuras anatómicas de la región. Normalmente, estas venas están próximas a la arteria braquial que, a nivel de la fosa cubital, se divide en las arterias ulnar y radial. Por tal motivo y, a pesar que recomendamos la utilización de la VMB, por su mayor calibre en el Tipo I, y la VMCo en el Tipo II, se debe tener presente que, profunda a ella, se encuentra la arteria braquial y el nervio mediano. Estas estructuras muchas veces están colocadas paralelas o se encuentran cubiertas totalmente por las venas, de ahí que para un buen acceso sea necesario extremar los cuidados. Asimismo, ramos anteriores del nervio cutáneo medial del antebrazo cruzan, generalmente la cara anterior de la VMB o de la VMCo y que, cuando son alcanzados, provocan un dolor agudo en dicha región.

A pesar que la distribución de las venas y los nervios cutáneos a nivel de la fosa cubital varían ampliamente, como lo señalan Yamada et al., y si bien ninguna zona es apta para todos los individuos, la punción de la VMCo cerca de la VCA es la menos probable de causar daño a los nervios. Por otra parte, del Sol \& Olave (1991) recomiendan utilizar preferencialmente, la VMB o VMCo recordando su estrecha relación con el nervio cutáneo medial del antebrazo, cuyos ramos la cruzan por sus parte anterior y posterior, como también su relación con la arteria braquial y nervio mediano. Si el calibre de la VCA o VMC es semejante al de la VMB o VMCo, es recomendable la utilización de ellas, ya que no tienen relaciones topográficas de importancia con elementos vasculares y nerviosos, siendo su relación más adyacente el nervio cutáneo lateral del antebrazo, que se ubica generalmente detrás de ellas.

En resumen, la disposición más frecuente según los estudios en los diferentes grupos étnicos no corresponde al esquema clásico de Winslow o M clásica, sino que esta M del codo está formada por la VCAA y la VBA, que forman en la cara ventral antebraquial las ramas venosas verticales lateral y medial, respectivamente, y la VCA que al bifurcarse forma las ramas oblicuas, a nivel de la fosa cubital y que confluyen hacia las VCA y VBA, para seguir como VB y VC en el brazo.

La VMA en general, es pequeña, termina drenando según el tipo de formación ya sea en la VMCo, en la unión de la VMB a la VBA o directamente en la VBA. Esta terminación de la VMA ayuda a aumentar el flujo de la VBA que después se continuará como VB en el brazo para formar, normalmente la vena axilar.

La presencia de la VCAA aunque no es constante, ella es muy frecuente y por tanto, se justifica su inclusión en la Terminología Anatómica. Esta vena se origina de la red venosa dorsal de la mano, constituyéndose como un tronco venoso en el tercio inferior de la superficie posterior del antebrazo, sigue con un trayecto oblicuo sobre el borde lateral del antebrazo, rodeando este borde se coloca ventralmente en el tercio superior. Así se une a la VMC en el Tipo I.

Finalmente, el abordaje de cualquier vena de la fosa cubital, para la extracción de sangre, administración medicamentosa, introducción de catéteres cardiacos, denudación venosa, entre otras, debe ser realizado con mucha precaución, teniendo presentes las observaciones antes señaladas y considerando además, las numerosas variaciones anatómicas presentes en la región cubital, especialmente la eventual presencia de arterias superficiales (del Sol et al., 2000).

Como señalaron Cagnon y Last una inyección intra o para-arterial accidental puede ocasionar desde un espasmo hasta una completa obstrucción, con secuelas tan importantes como la amputación del segmento afectado, lo que adquiere importancia al utilizar las venas superficiales de la fosa cubital. 
DEL SOL, M. \& VÁSQUEZ, B. Superficial veins of the cubital fossa. Anatomical, clinical and anthropological aspects. Int. J. Morphol., 27(2):527-538, 2009.

SUMMARY: Access of the cubital fossa to the superficial veins is very frequent, this being one of the most frequent vein puncture sites. Although it is a simple procedure, it is invasive and at times painful. The disposition of the superficial veins of the cubital area has been described by numerous authors and in diverse ethnic groups, describing many variations and various patterning. The superficial veins of the of the cubital fossa have been independently described, forming an M, N, Y or W. Numerous studies in different races and ethnic groups have demonstrated similarities and differences in the disposition of the superficial veins of the cubital fossa. In 1908 Berry \& Newton determined that in $83 \%$ of British men the cephalic vein of the forearm (CVF) and the basilic vein of the forearm (BVF) were connected by the median cubital vein (MCV) Okamoto (1922) in Japanese men, determined 3 types of venous patterns. Type I where the CVF originates the MCV, the accessory cephalic vein (ACV) does not exist, and the (CVF) does not duplicate; Type II is characterized by the duplication of the CVF and a Type III, that includes the ACV which drains in the CVF. In white and black men Charles (1932) indicated that the most frequent distribution (nearly 70\% of the cases), was that where the CVF and the BFV were connected by the MCV. Soller et al. (1962, 1964) in Africans from West Africa, distinguished three types of venous formations. Group I classic dispositions, types in M or apparent (38.1\%) Groups II and III described as dispositions of the embryological type constitute $62 \%$ of the cases. Halim \& Abdi (1974) observed 3 types in Hindus types: 1) Type I. The CVF and the BVF are connected by the MCV; Type II. The CVF drains in the BVF: the median vein of the forearm (MVF) drains in the CVF. Type III. There is no communication between the CVF and the BFV in the cubital fossa and it is subdivided in Types III A and III B. Wasfi et al. (1986) described 6 types of venous formations in Iraqis, indicating that two of them had not been mentioned previously. The most common type was the division of the MVF in two veins, one of which joined to the BVF and the other to the CVF. Del Sol et al. (1988) classified the venous formations of the cubital fossa in 5 Types: I the CVF divides into median basilic vein (BMV) and median cephalic vein (MCV), joining the BVF and ACV respectively. Type II, the CVF originates vein MCV which joints BVA, ACV does not exist. Type III there is no communication between BVF and CVF at the level of the cubital fossa, Type IV the CVF drains in the BVF and the MVF drains in the CVF. Type V other dispositions, where the classic M is included which results from the MVF division. With respect to the diameter of the veins it appears to be the consensus that depending on the type of formation of the MVF and the $\mathrm{MCB}$ these are of the greater diameter. The use of the MCV is recommended when it presents a similar diameter to the MBV or the MCV since the risks of puncture to other anatomical structures, such as anterior branches of the medial cutaneous nerve of the forearm or brachial artery are minimal.

KEY WORDS: Anatomy; Veins; Cubital fossa; Vein puncture.

\section{REFERENCIAS BIBLIOGRÁFICAS}

Bardeleben apud Poirier, P. \& Charpy, A. 1902, op. cit.

Berry, R. J. A. \& Newton, H. A. S. A study of the superficial veins of the superior extremity in 300 living subjects. Anat.Anz., 33(5):591-601, 1908.

Bertelli apud Poirier, P. \& Charpy, A. 1902, op. cit.

Cagnon, R. Superficial arteries of the cubital fossa with reference to accidental intra-arterial injections. Can. J. Surg., 9:5765,1966 .

Charles, C. M. On the arrangement of the superficial veins of the cubital fossa in American white and American negro males. Anat. Rec, 54:9-14, 1932.

Chiarugi, G. Istituzioni di Anatomía dell'Uomo. Milano, Societa Editrice Libraría, 1912. V. 2.

Comité International de la Nomenclature Anatomique. Nomina Anatómica. Ballantyne, Londres, 1985. (Imprimé a titre de publication privée), 2000.

Del Sol, C. M.; Aguilar, M. A,; Collipal, E.; Mandioca, L. E. \& Olave R. E. Arteria radial superficial en la fosa cubital: importancia anátomo-clínica. Rev. chil. cienc. méd. biol., 5(1):27-33, 1995.
Del Sol, M.; DeAngelis, M. A. \& Bolini, P. A. D. Formações venosas na fossa cubital da criança. Pediatría Moderna, 23(4):225-31, 1988.

Del Sol, M.; Mardones, L. M. \& Bustos, T. E. Formaciones Venosas de la Fosa Cubital en el Individuo Mapuche. Estudio Bioscópico. Int. J. Morphol, 25(4):885-94, 2007.

Del Sol, M. \& Olave, E. Venas de la fosa cubital en el hombre. Sitios de punción. Rev. Chil. Cs Med.Biol., 1(1):49-53, 1991.

Del Sol, M.; Olave, E.; Hettich, M. E. \& Bustos, K. Arteria ulnar superficial: importancia anátomo-clínica. Rev. Chil. Anat., 18(1):2000.

Del Sol, M.; Wuster, A. \& Fritz, I. Tipos de formaciones venosas en la fosa cubital en jóvenes chilenos. Rev. Chil. Tecnol. Méd., 13(2):646-9, 1990.

Duval, M. apud Poirier, P \& Charpy, A. 1902, op. cit.

Federative Committee Anatomical Terminology (FCAT). Terminología Anatómica. International Anatomical Terminology. Thieme, New York, 2000. 
Fumagalli, Z. \& Cavalloti, C. Anatomia umana normale. Roma, Piccin, 1982. V. 1.

Halim, A. \& Abdi, S. H. M. Superficial venous patterns in the cubital region of Indians. Anat. Rec., 178(3):631-6, 1974.

Hamilton, W. J. Tratado de anatomia humana. Rio de Janeiro, Interamericana, 1977.

Henckel, C. Antropología Física de los mapuches. Rev. Universitaria, 43(22):13-22, 1958.

International Anatomical Nomenclature Committee. Nomina Anatómica. $6^{\text {th }}$. ed. Edinburgh, Churchill Livingstone, 1989.

Jamain, A. Nuevo Tratado de Anatomía Descriptiva. Cárlos BaillyBailliere, Madrid, 1862.

Lagos, M. M. \& Torres, B. E. Formaciones venosas de la fosa cubital en el individuo mapuche. Seminario de investigación, Universidad de La Frontera, 1991.

Last, R. J. Superficial veins of the forearms. The surgical anatomy in relation to intravenous injection. Brit. Dental J., 125(11):4914, 1968.

Lassila apud Loth, E. Anthropologie des partes molles. Varsovia \& Paris, 1931. op cit.

Latarjet, M. \& Ruiz Liard, A. Anatomía Humana. Panamericana, Buenos Aires, 1983. V. 1.

Moore, K. L. Anatomía. Buenos Aires, Panamericana, 1982.

Okamoto, K. A study of the superficial veins in the superior extremity of live Japanese. Anat. Rec., 23:323-33, 1922.

Orts Llorca, F. Anatomía humana. Barcelona, Científica Médica, 1952.V. 3.

Paturet, G. Traite d'anatomie humaine. Paris, Masson, 1951. V. 2.

Pernkopf, E. Anatomía topográfica. Barcelona, Labor, 1953. V. 1.

Poirier, P. \& Charpy, A. Traite d'anatomie humaine. 2. ed.Paris, Masson, 1902. V. 3.

Queiroz, G. F. Tratado de Anatomía Humana. Porrua, México, 1993. V. 2.

Rouviére, H. Anatomie humaine. Paris, Masson, 1924. V.2.

Salvi, G. Angiología in Balli, R.; Bertelli, D. et al. Trattato di anatomía umana. 2a. ed. Milano, Casa Editrice Dottor Francesco Vallardi, 1932. V.3.

Sappey, Ph. Traite d'anatomie descriptive. $3^{\mathrm{a}}$. ed. París, Adrien Delahaye, 1876. V. 2.
Saunders, J. B. DeC. M. \& O'malley, C D. Andreas Vesalius de Bruxelas. De humani Corporis Fabrica. Epitome. Tabulae Sex. Ateliè Editorial, Campinas, 2002.

Singh, S. P.; Ekandem, G. J. \& Bose, E. S. A study of the superficial veins of the cubital fossa in Nigerian subject. Acta Anat., 774:311-20, 1982.

Skewes, E. Estudio de las venas superficiales del antebrazo en los chilenos. Bol. Soc. Biol., 19:75-81, 1944.

Snell, R. S. Anatomia. $2^{\mathrm{a}}$ ed. Rio de Janeiro, MEDSI, 1984.

Sohier, H. M. L.; Fustec, R. \& Laffont, J. Veines superficielles du pli du coude chez 1'Africain d'Afrique occidental. Bull. Soc. Méd.. AfriqueNoire., 7(7):107-11, 1962.

Sohier, H. M. L.; Fustec, R.; Laffont, J. \& Le Guyader, A. Veines superficielles du pli du coude chez Y Africain d'Afrique occidental. Bull Assoc. Anatomistes., 720:1230-5, 1964.

Smith, H. H. Anatomical atlas, ilustrative of the structure of the human body. Lea and Blanchard, Philadelphia, 1845.

Tandler, J. Tratado de anatomía sistemática. Barcelona, Salvat, 1929.

Testut, L. Trattato di anatomía umana. Torino, Unione tipográficoEditrice, 1894.V.2.

Thiele apud Poirier, P \& Charpy, A. 1902, op. cit.

Tillaux, P. Traité d'anatomie topographique avec applications a la chirurgie. $10^{\mathrm{a}}$ ed. Asselin \& Houzeau, Paris, 1900.

Warwick, R. \& Williams, P. L. Gray-Anatomia. 35a. ed. Rio de Janeiro, Guanabara Koogan, 1979. V. 1.

Wasfi, F.A.; Dabbagh, A.W.; AlAthari, F.M. \& Salman, S.S. .Biostatistical Study on the Arrangement of the Superficial Veins of the Cubital Fossa in Iraqis. Acta Anatomica, 126:183-6, 1986.

Winslow apud Poirier, P \& Charpy, A. 1902, op.cit.

Woodburne, R.T. Anatomía humana. 6a. ed. Rio de Janeiro, Guanabara Koogan, 1984.

Yamada, K.; Yamada, K.; Katsuda, I. \& Hida , T. Cubital fossa venipuncture sites based on anatomical variations and relationships of cutaneous veins and nerves. Clin. Anat., 21:30713, 2008.

Dirección para correspondencia:

Prof. Dr. Mariano del Sol

Facultad de Medicina

Universidad de La Frontera

Casilla 54-D

Temuco - CHILE

Email: mdelsol@ufro.cl

Recibido : 22-01-2009

Aceptado: 20-03-2009 